\title{
共聚焦显微术测定大鼠和黄鼠心肌细胞 游离 $\mathrm{Ca}^{2+}$ 浓度与温度的关系"
}

\author{
王世强 周曾铨 \\ (北京大学生命科学学院, 北京 100871) \\ 钱 洪 \\ (中国农业科学院蔬菜花卉研究所, 北京 100081)
}

\begin{abstract}
摘要 运用共聚焦激光扫描显微成像术对比研究非冬眠动物大鼠和冬眠动物黄鼠 心肌细胞胞内 $\mathrm{Ca}^{2+}$ 浓度 $\left(\left[\mathrm{Ca}^{2+}\right]_{\mathrm{i}}\right)$ 随温度的变化. 首先标定了不同温度下 $\mathrm{Ca}^{2+}$ 探 针 indo 1 的解离常数, 提出并证明按 $\alpha$ 定态设定标定溶液 $\mathrm{pH}$ 值的必要性. 细胞荧 光分析显示, 大鼠心肌细胞 $\left[\mathrm{Ca}^{2+}\right]_{\mathrm{i}}$ 随温度降低显著上升, 低温下频繁出现自发钙 波, 胞内发生钲超载; 相比较冬眠动物黄鼠心肌细胞 $\left[\mathrm{Ca}^{2+}\right]_{\mathrm{i}}$ 在相同条件下保持稳定, 避免发生铨超载. 认识其中的钘稳态机制可能对有关医学问题有潜在的指导意义.
\end{abstract}

\section{关键词 心肌细胞 钻稳态 冬眠 温度 共聚焦激光扫描显微成像术 $\mathrm{Ca}^{2+}$ 荧光探针}

人和许多非冬眠哺乳动物心脏保持节律收缩的最低温度为 $20 \sim 28^{\circ} \mathrm{C}$. 心温的降低通常 会导致心室纤㗜 心搏停止以及其他严重的心律失常, 这是低温麻醉和器官移植等临床医学 领域所遇到的问题. 然而, 冬眠哺乳动物体温在 $0 \sim 37^{\circ} \mathrm{C}$ 之间变动并无生命之忧, 心温接近 冰点仍能维持洜血功能. 探讨两类哺乳动物心肌机能差异不仅是一个动物学问题, 也将为解 决有关医学问题提供思路.

离体研究表明, 非冬眠哺乳动物心室乳头肌在 $15^{\circ} \mathrm{C}$ 以下舒期张力增加, 并出现后收缩现 象, 最后失去收缩性 ${ }^{[1]}$; 电生理研究也证明低温下大鼠心肌细胞瞬时内向电流增加 ${ }^{[2]}$. 上述 现象提示低温下胞内发生钙超载( calcium overload). 冬眠哺乳动物心肌能在低温下保持有力 的收缩, 不出现舒期张力上升和后收缩的现象 ${ }^{[1]}$, 意味着其细胞能在低温下维持钻稳态, 避 免钲超载. 上述推论的检验, 有赖于胞内 $\mathrm{Ca}^{2+}$ 浓度 $\left(\left[\mathrm{Ca}^{2+}\right]_{\mathrm{i}}\right)$ 的测定.

目前, $\mathrm{Ca}^{2+}$ 荧光探针已广泛用于 $\left[\mathrm{Ca}^{2+}\right]_{\mathrm{i}}$ 测定. 由于这些探针的 $\mathrm{Ca}^{2+}$ 解离常数 $\left(K_{\mathrm{d}}\right)$ 与温 度、离子强度和 $\mathrm{pH}$ 值有关 ${ }^{3]}$, 因此在不同温度下使用 $\mathrm{Ca}^{2+}$ 探针需要充分考虑这些因素. 细 胞内, 温度每降低 $1{ }^{\circ} \mathrm{C}, \mathrm{pH}$ 值约下降 0.016 , 这主要与蛋白质组氨酸残基 $\alpha$ 咪唑环对 $\mathrm{pH}$ 的缓 冲有关 ${ }^{[4]}$. 低体温研究中将维持组氨酸 $\alpha$ 咪唑环解离程度不变的 $\mathrm{pH}$ 设定称为 $\alpha$ 定态, 相应 地, $\mathrm{pH}$ 在不同温度下设成定值称为 $\mathrm{pH}$ 定态 ${ }^{[5]}$. 在不同温度下测定 $\left[\mathrm{Ca}^{2+}\right]_{\mathrm{i}}$ 时, 标定 $K_{\mathrm{d}}$ 时 $\mathrm{pH}$ 
值应与胞内 $\mathrm{pH}$ 同步变化, 即符合 $\alpha$ 定态. 但这种考虑迄今尚未见报道, 其必要性有待探讨.

关于心肌细胞 $\left[\mathrm{Ca}^{2+}\right]_{\mathrm{i}}$ 与温度的关系, 目前唯有 $\mathrm{Liu}$ 等人用 $\operatorname{indo} 1$ 荧光分光光度法进行的 测定 ${ }^{[6]}$. 但其中不同温度的 $K_{\mathrm{d}}$ 均在 $\mathrm{pH} 7.0$ 下标定. 温度越低, 细胞内 $\mathrm{pH}$ 值越远离 7.0, $K_{\mathrm{d}}$ 偏离实际值也越大, 这降低了他们所得结果的可信度. 因此, 低温下心肌细胞 $\left[\mathrm{Ca}^{2+}\right]_{\mathrm{i}}$ 究竟 如何变化, 尤其是冬眠动物是否有特殊之处, 仍有待研究.

本文将共聚焦激光扫描显微成像技术应用于解决上述问题. 选择 indo- 1 为 $\mathrm{Ca}^{2+}$ 探针, 首 先证明 $\alpha$ 定态下标定 $K_{\mathrm{d}}$ 的必要性, 然后在此基础上对比测定了非冬眠动物大鼠和冬眠动物 黄鼠心肌细胞 $\left[\mathrm{Ca}^{2+}\right]_{\mathrm{i}}$ 随温度的变化, 发现冬眠动物在维持细胞钙稳态方面具有特殊适应性.

\section{1 材料与方法}

\section{1 实验动物}

实验采用体重约 $200 \mathrm{~g}$ 的成年 Wistar 大鼠和体重 200 300 g 的成年黄鼠( Citellus dauricus), 雌雄不拘. 其中黄鼠自山西阳高野外捕到, 饲养于动物房至少习服 1 个月, 取材时 动物清醒, 体温 $35 \sim 37^{\circ} \mathrm{C}$.

\section{2 心肌细胞的分离}

实验动物以乙醚麻醉, 迅速开胸取心, 连接到 Langendorff 灌流系统, 以无钲生理溶液 $37^{\circ} \mathrm{C}$ 恒温灌流. 无钙生理溶液的成分为 $(\mathrm{mmol} / \mathrm{L}): 110 \mathrm{NaCl}, 4 \mathrm{KCl}, 1.2 \mathrm{MgCl}_{2}, 1.2 \mathrm{NaH}_{2} \mathrm{PO}_{4}, 20$ $\mathrm{NaHCO}_{3}, 30$ 牛磺酸, 10 葡萄糖, 通以 $95 \% \mathrm{O}_{2}+5 \% \mathrm{CO}_{2}, \mathrm{pH} 7.4$. 无钙灌流 $5 \mathrm{~min}$ 后改灌含 $0.5 \mathrm{mg} / \mathrm{mL}$ 胶原酶(Sigma 1A 型), $1 \mathrm{mg} / \mathrm{mL}$ 牛血清白蛋白( Sigma, 组分 $\mathrm{V}$, 无脂肪酸型) 和 75 $\mu_{\mathrm{mol} / \mathrm{L} \mathrm{CaCl}}$ 的生理溶液. 约 $15 \mathrm{~min}$ 后心脏松软, 取心室组织剪碎, 在酶液中保温 $10 \mathrm{~min}$, 经 过通氧轻轻吹动. 所得细胞悬液滤至 10 倍体积含 $0.2 \mathrm{mmol} / \mathrm{L} \mathrm{CaCl}_{2}$ 的生理溶液静置, 细胞沉 淀于管底. 通过将细胞沉淀分散再沉淀, 逐步将胞外 $\left[\mathrm{Ca}^{2+}\right]$ 提高到 $2 \mathrm{mmol} / \mathrm{L}$. 最后细胞被储 存于如下生理溶液 (mmol/L) : $140 \mathrm{NaCl}, 4 \mathrm{KCl}, 2.0 \mathrm{CaCl}_{2}, 1.2 \mathrm{MgCl}_{2}, 1.2 \mathrm{NaH}_{2} \mathrm{PO}_{4}, 10$ glucose, 5 HEPES, 用 $\mathrm{NaOH}$ 调至 $\mathrm{pH}$ 7. 4. 实验中观察到杆状健康细胞沉降速度较快, 因此 2 3 次的 沉淀分散过程富集了健康细胞, 细胞沉淀呈肉红色, 杆状细胞比例为 60\% 90\%。

\section{3 细胞荧光染色}

事先先将 indo-1 AM (Molecular Probe Inc. ) 配成 $1 \mu_{\mathrm{g}} / \mu_{\mathrm{L}}$ 的二甲亚砜( Sigma，纯度 99.9\%) 溶液, 分装后- $20^{\circ} \mathrm{C}$ 保存备用. 染色前将 $2.5 \mu_{\mathrm{g}}$ indo $1 \mathrm{AM}$ 溶于 $1 \mathrm{~mL}$ 生理溶液. 加入 $100 \mu \mathrm{L}$ 细胞悬液, 混合均匀, $37^{\circ} \mathrm{C}$ 保温 $15 \mathrm{~min}$ 后以生理溶液冲洗. 上述操作避光进行.

\section{4 标本槽及温度控制}

盛放待测细胞的标本槽由塑制培养血改制，皿底中心 $1 \mathrm{~cm}$ 直径的部分换为盖玻片，目的 是与显微镜物距相适应. 皿内中央粘置环形玻璃管, 与 Cole-Parmer 低温循环水浴相连, 用以 控制温度. 环形玻璃管围成容积约 $0.5 \mathrm{~mL}$ 的小室用于盛放溶液和细胞. 电子温度计的探头 紧贴血底放置, 用以监视温度. 当盛有 $0.3 \mathrm{~mL}$ 以上溶液时, 小室底部中心与周边温差小于 $0.5^{\circ} \mathrm{C}$.

\section{5 荧光图像的记录和 $\left[\mathrm{Ca}^{2+}\right]_{\mathrm{i}}$ 的计算}

荧光图像由 ASCS Meridian 575UV 共聚焦激光扫描显微成像系统记录. 硬件设置为 (商品 
名词不加翻译): UV 激光光源, $40 \times \mathrm{UV}$ 物镜, UV only tower cube, indo- 1 专用镜组. 固定的软 件设置为 ratio/ calcium/ image 单元, PMT1 55\%, PMT2 50\%。其中 PMT1 和 PMT2 分别是针对 485 和 $405 \mathrm{~nm}$ 波长苂光信号的检测通道. 激光光源的功率和通透比率、扫描的速度和范围根 据记录需要进行调整.

数据处理时通过 region query 测得细胞区域 PMT2/ PMT1 比值 FR, 依下式 ${ }^{[7]}$ 求得 $\left[\mathrm{Ca}^{2+}\right]_{\mathrm{i}}$ :

$$
\left[\mathrm{Ca}^{2+}\right]=K_{\mathrm{d}} \frac{\mathrm{FR}-\mathrm{FR}_{\min }}{\mathrm{FR}_{\max }-\mathrm{FR}} \cdot \beta,
$$

其中 $\mathrm{FR}_{\text {max }}$ 和 $\mathrm{FR}_{\text {min }}$ 分别是饱和钙和无钙溶液的 $\mathrm{FR}$. $\beta$ 为波长 $480 \mathrm{~nm}$ 的信号在饱和钙和无钙 溶液中的比值.

\section{6 indo $1 K_{d}$ 的测定}

测定 indo- $1 K_{\mathrm{d}}$ 所用钙缓冲液成分如下: $140 \mathrm{mmol} / \mathrm{L} \mathrm{KCl}, 1 \mathrm{mmol} / \mathrm{L} \mathrm{EGTA,} 25 \mathrm{mmol} / \mathrm{L}$ HEPES, $2 \mu_{\mathrm{mol}} / \mathrm{L} \mathrm{K}_{5}$ indo- 1 (Sigma), 以及含量不等的 $\mathrm{CaCl}_{2}$. $\mathrm{pH}$ 值由 $\mathrm{KOH}$ 调节, 其中 $\mathrm{pH}$ 定态 下调至 7. 2, $\alpha$ 定态下按照表 1 调节. 缓冲液中 $\left[\mathrm{Ca}^{2+}\right]$ 的计算及所涉及常数的温度和离子强度 校正参照 Harrison 和 Bers ${ }^{[8]}$ 的方法. 实验测定 $\mathrm{FR}_{\max }, \mathrm{FR}_{\min }, \beta$ 以及各 $\left[\mathrm{Ca}^{2+}\right]$ 相应的 FR. 所得 数据由(1) 式进行拟合求得 $K_{\mathrm{d}}$.

表 1 本文设定的 $\alpha$ 定态 $\mathrm{pH}$ 值 ${ }^{\mathrm{a}}$

\begin{tabular}{ccccccc}
\hline 温度/ ${ }^{\circ} \mathrm{C}$ & 35 & 30 & 25 & 20 & 15 & 10 \\
\hline $\mathrm{pH}$ & 7.02 & 7.10 & 7.18 & 7.27 & 7.36 & 7.45 \\
\hline
\end{tabular}

a) 表列值按组氨酸残基 $\alpha$ 咪唑环 $\mathrm{H}^{+}$解离为焓变 $\Delta H=7 \times 4.1868 \mathrm{~kJ} / \mathrm{mol}^{[4]}$ 计算得到

由于细胞蛋白对 $\mathrm{Ca}^{2+}$ 与荧光探针的结合有显著影响, 最后用于计算细胞 $\left[\mathrm{Ca}^{2+}\right]_{\mathrm{i}}$ 的 $K_{\mathrm{d}}$ 在 细胞原位标定. 先取少量大鼠心肌细胞在成分为 $140 \mathrm{mmol} / \mathrm{L} \mathrm{KCl}, 1 \mathrm{mmol} / \mathrm{L} \mathrm{EGTA}, 25 \mathrm{mmol} / \mathrm{L}$ HEPES, $0.5 \%$ Triton X-100 的溶液中通透化, 然后将少量细胞悬液滴入标本槽, 小心吸去溶液, 加入待测 indo- 1 溶液, 其中 EGTA 增加到 $10 \mathrm{mmol} / \mathrm{L}$ 以消除细胞内钲缓冲体系对 EGTA 缓冲效 果的影响. 实验测定细胞区域的苂光数据, 由(1) 式拟合求得 $K_{\mathrm{d}}$.

\section{7 数据的统计处理}

实验数据统计后表示为平均值士标准误. 数据图绘制和曲线拟合借助 Sigma Plot 软件完 成, 拟合度以相关系数 $r$ 表示; 统计检验由 Sigma Stat 软件进行, 使用有重复试验的单因素或双 因素方差分析模型, $p<0.05$ 认为显著, $p<0.01$ 认为极显著.

\section{2 结果}

\section{$2.1 K_{d}$ 与温度的关系测定}

$\mathrm{Z}$ 在一定 $\mathrm{pH}$ 值和温度下, 随着 $\left[\mathrm{Ca}^{2+}\right]$ 增加, indo- 1 溶液在 PMT1 信号下降, PMT2 信号增 加, 从而 FR 增加, 其变化曲线呈 $\mathrm{S}$ 形. 用(1) 式对 $\mathrm{FR}\left[\mathrm{Ca}^{2+}\right]$ 曲线进行拟合, 得到不同温度和 $\mathrm{pH}$ 下的 $K_{\mathrm{d}}$. 图 1 显示了 $\mathrm{pH} 7.2$ 和 $\alpha$ 定态两种情况下 $K_{\mathrm{d}}$ 随温度的变化. $\mathrm{pH} 7.2$ 下, 当温度由 $35^{\circ} \mathrm{C}$ 降到 $10{ }^{\circ} \mathrm{C}, K_{\mathrm{d}}$ 从(212.1 士3.4) nmol/L 增加到( 280. 2 土4. 6) nmol/ L, 说明 indo- 1 与 $\mathrm{Ca}^{2+}$ 的亲和性随温度降低而下降. 然而 $\alpha$ 定态下 $K_{\mathrm{d}}$ 变化显著较小.

indo- $1 K_{\mathrm{d}}$ 的原位标定在 a 定态下进行. 测定中观察到, 共聚焦系统每个 PMT 在细胞区域 
检测到的信号总比胞外区域强 2 3 倍, 其比值 FR 也与胞外区域不同. 这与文献 $[9,10]$ 报道一致, 说 明细胞成分对 indo 1 苂光的动力学有影响.

\section{2 心肌细胞 $\left[\mathrm{Ca}^{2+}\right]_{i}$ 随温度的变化}

实验得到大鼠和黄鼠心肌细胞不同温度下的 indor 1 荧光图像. 图版 I-1 ( 附本刊后, 下同) 为一 例典型结果, 显示大鼠心肌细胞 FR 随温度降低明 显增加. 在温度下降过程中, 特别是约 $15^{\circ} \mathrm{C}$ 以下, 常在显微镜中观察到大鼠心肌细胞自发收缩. 与 刺激驱动下细胞整体收缩不同, 自发收缩从细胞一 端开始蚓动状传向另一端, 其胞内钲过程被称为钙 波( calcium wave). 图版 I-2 显示了 1 例在 $14{ }^{\circ} \mathrm{C}$ 捕 获的大鼠心肌细胞钙波, 其中还分辨出可能为钙火 花( calcium spark $)^{[11]}$ 的“小峰”. 在钙波前沿“小峰” 较多, 钙波传过之后, “小峰” 暂时减少, $\left[\mathrm{Ca}^{2+}\right]_{\mathrm{i}}$ 本

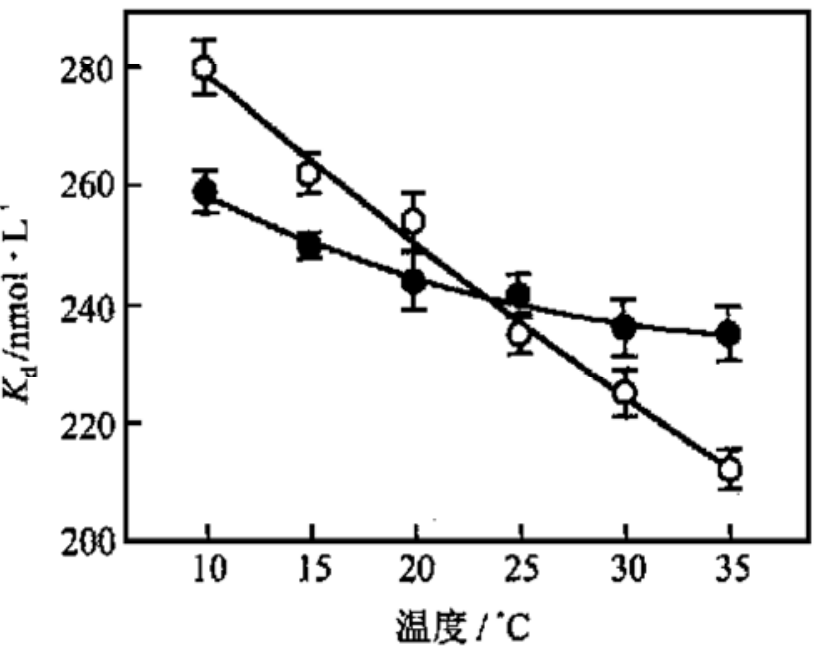
底水平也先暂有下降, 然后逐渐回升, “小峰”也逐渐增多, 一定时间后发生另一次钲波. 我 们注意到，频繁发生钙波的心肌细胞若不及时复温，细胞逐渐缩成球状，此状态不可恢复.

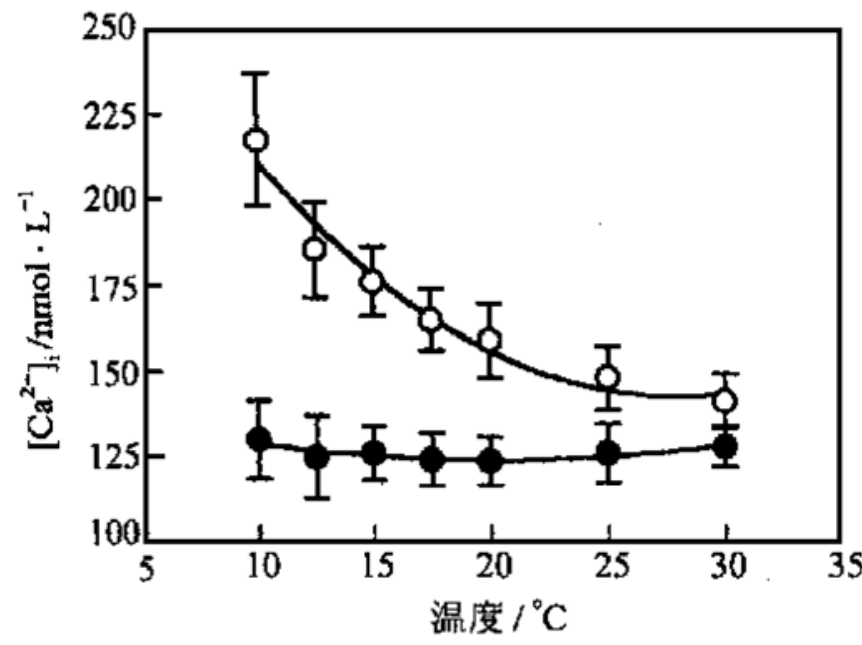

黄鼠的情形与大鼠很不相同. 图版 II 显示黄 鼠心肌细胞 FR 随温度没有明显变化, 在 $10{ }^{\circ} \mathrm{C}$ 左 右也能保持比较稳定的状态, 很少记录到自发钙 波, 也很少观察到自发收缩.

对所得细胞荧光图像进行分析计算得到 $\left[\mathrm{Ca}^{2+}\right]_{i}$. 由图 2 可见大鼠细胞 $\left[\mathrm{Ca}^{2+}\right]_{\mathrm{i}}$ 随温度降 低呈指数增加, 从 $30^{\circ} \mathrm{C}$ 的 $(141 \pm 8) \mathrm{nmol} / \mathrm{L}$ 增加 到 $10{ }^{\circ} \mathrm{C}$ 的 $(218 \pm 12) \mathrm{nmol} / \mathrm{L}$; 而黄鼠则维持在 130 $\mathrm{nmol} / \mathrm{L}$ 附近.

图 2 大鼠 ( O, $n=12)$ 和黄鼠 $(\bullet$,

3 讨论

$n=8)$ 心肌细胞内钙离子浓度随温度的变化 单因素方差分析显示温度对 $\left[\mathrm{Ca}^{2+}\right]_{i}$ 的影响在大鼠组极

显著 $(p<0.01)$, 但在黄鼠组不显著 $(p>0.05)$

\section{1 选择 indo 1 为荧光探针}

80 年代以来, Tsien 及其同事发展的 $\mathrm{Ca}^{2+}$ 苂 光探针 ${ }^{[7]}$ 大大方便了 $\left[\mathrm{Ca}^{2+}\right]_{i}$ 的测量. 目前常用 的 $\mathrm{Ca}^{2+}$ 荧光探针有 indo- 1 , fura 2 , fluo 3 等. 其中 fluo 3 是较新一代探针, 量子效率较高, 且用 蓝光激发, 对细胞损伤小, 目前应用较多. 但本研究采用 indo-1 更为可行. (1) 式中 $\left[\mathrm{Ca}^{2+}\right]$ 的 计算采用 2 个波长荧光的比值, 从而尽可能消除了探针的浓度和分布差异, 光漂白对测量的 影响; 更重要的是 $\mathrm{FR}_{\text {max }}, \mathrm{FR}_{\text {min }}$ 和 $\beta$ 在相同的温度和仪器设置下是常数, 可以与 $\mathrm{FR}$ 分别测量, 这使不同温度条件下连续观测同一细胞成为可能.

indo 1 的弱点是紫外光源激发, 容易损伤细胞, 标志是 $\left[\mathrm{Ca}^{2+}\right]_{\mathrm{i}}$ 迅速增加, 细胞痉挛, 并 很快缩成球状. 本文实验设计中充分考虑了紫外损伤的防护：(1) 尽量降低光源能量和通透 
比率，这样需提高 PMT 倍率，但 PMT 倍率太高会助长噪声，本文 PMT 设为 50\% 60\%；(2) 在兼顾图像分辨率的情况下尽量增加扫描速度, 降低采样频率; (3) 在兼顾信噪比的同时尽 量减少采样点的迭加平均次数; (4) 尽量缩短测量时间, 一次扫描在 $5 \mathrm{~s}$ 内完成; (5) 对所用 参数进行可靠性检验, 标准是延长测量时间若干倍, 细胞 FR 仍能保持稳定.

\section{2 关于 $\mathrm{Ca}^{2+}$ 荧光探针的标定方法}

由于荧光探针的 $K_{\mathrm{d}}$ 对温度、离子强度和 $\mathrm{nH}$ 值的敏感性 ${ }^{[3]}$. 研究不同温度下 $\left[\mathrm{Ca}^{2+} 1_{\mathrm{i}}\right.$ 的变 化, 应充分考虑这些因素. 本文发现, $\alpha$ 定态下 $K_{\mathrm{d}}$ 的温度依赖性比在 $\mathrm{pH}$ 定态下小很多, 这说 明温度增加引起的 $K_{\mathrm{d}}$ 减小会被细胞 $\left[\mathrm{H}^{+}\right]$增加引起的副效应所缓冲. 对比图 2 和图 1 可见, 温度对 $\left[\mathrm{Ca}^{2+}\right]_{\mathrm{i}}$ 的影响和 $\mathrm{pH}$ 设定方式对 $K_{\mathrm{d}}$ 的影响在相同的数量级上, 这说明, 要用 $K_{\mathrm{d}}$ 计算 $\left[\mathrm{Ca}^{2+}\right]_{\mathrm{i}}, \mathrm{pH}$ 随温度变化所带来的误差不可忽略. 因此, 我们认为, 当 indo- 1 等 $\mathrm{Ca}^{2+}$ 荧光探 针在不同温度下使用时，应当在 $\alpha$ 定态下进行标定. 以往有关研究在 $\mathrm{pH}$ 定态下进行标定, 所得 $\left[\mathrm{Ca}^{2+}\right]_{\mathrm{i}}$ 随温度变化的结果都存在系统误差.

由于蛋白质环境对 $\mathrm{Ca}^{2+}$ 荧光探针的解离性质有影响 ${ }^{[9,10]}$, 用于计算 $\left[\mathrm{Ca}^{2+}\right]_{\mathrm{i}}$ 的 $K_{\mathrm{d}}$ 需要在 近似细胞内的有机环境或细胞原位进行标定. 文献 [9] 所用方法有的是在标定液中加入肌肉 蛋白, 有的用 A23187 使载有 indo- 1 的细胞对 $\mathrm{Ca}^{2+}$ 通透化 ${ }^{[10]}$, 还有更为复杂的方法. 本文是 在 indo- 1 溶液中加入用 Triton X-100 通透化的细胞, 直接测量. 由于共聚焦系统只能记录焦平 面的荧光，所以系统扫描于细胞内时只能记录到来自细胞的信号. 此法比以往方法都简单, 尚未见报道. 事实上, 运用共聚焦系统, 无机环境标定和细胞原位标定可以同时进行, 因为 细胞区域和细胞外区域可以扫在一幅图像中.

\section{3 低温下大鼠心肌细胞发生钙超载}

本文测得大鼠心肌细胞 $\left[\mathrm{Ca}^{2+}\right]_{\mathrm{i}}$ 由 $30^{\circ} \mathrm{C}$ 的 $(141 \pm 8) \mathrm{nmol} / \mathrm{L}$ 增加到 $10{ }^{\circ} \mathrm{C}$ 的 $(218 \pm 12)$ $\mathrm{nmol} / \mathrm{L}$. 由于我们比 $\mathrm{Liu}$ 等人 ${ }^{[6]}$ 在 $K_{\mathrm{d}}$ 标定和研究手段上有进一步的设计, 因此我们的结果较 确業地说明了非冬眠动物心肌细胞 $\left[\mathrm{Ca}^{2+}\right]_{\mathrm{i}}$ 随温度的变化规律.

$\left[\mathrm{Ca}^{2+}\right]_{\mathrm{i}}$ 相对稳定是细胞正常生理活动的前提. [ $\left.\mathrm{Ca}^{2+}\right]_{\mathrm{i}}$ 过度升高不仅妨碍线粒体供给能 量的能力, 也会造成细胞膜系统损伤. 在心肌细胞, $\left[\mathrm{Ca}^{2+}\right]_{\mathrm{i}}$ 过高会导致舒张不利, 引起收缩 性下降甚至丧失 ${ }^{[2]}$; 还会导致肌质网钲致钻释放的自激振荡, 伴随着瞬时内向电流的周期性 激活, 在力学上表现为后收缩, 在电学上表现为迟后去极化, 引起心律失常 ${ }^{[13]}$. 此时, $\left[\mathrm{Ca}^{2+}\right]_{\mathrm{i}}$ 超过正常生理限度, 发生了钲超载. 本文所发现大鼠心肌细胞低温下自发钙波的频 繁发生, 以及我们以往观察到低温下的后收缩现象 ${ }^{[1]}$ 都是上述过程的体现. 可见, 大鼠心肌 细胞在低温下 $\left[\mathrm{Ca}^{2+}\right]_{\mathrm{i}}$ 升高，已导致细胞功能障碍，表明了胞内钻超载的发生.

胞内钙超载的发生有两方面可能性：一是胞外进入过多，二是胞内移除不力. 对静息的 心肌细胞来说, 后者可能是主要因素. 究竟是哪些机制导致低温下钘超载还有待研究. 可以 预期，低温下有关酶促过程减慢甚至停止是其中的重要原因.

\section{4 黄鼠心肌细胞钻稳态调节的适应性}

与大鼠的情形迥然不同, 我们发现冬眠动物黄鼠心肌细胞在温度变化过程中保持 $\left[\mathrm{Ca}^{2+}\right]_{\mathrm{i}}$ 基本稳定, 体现了其对低体温的适应性. 这与其肌条舒期张力的稳定和不出现后收缩的现

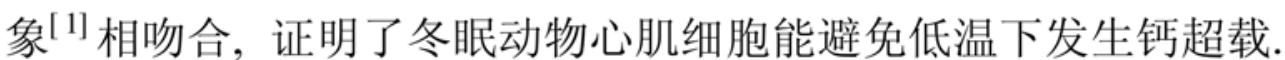


冬眠动物避免细胞钙超载的发生, 说明其有效地解决了非冬眠动物所遇到的问题, 使胞 浆 $\mathrm{Ca}^{2+}$ 在低温下也能有效移除. 目前关于冬眠动物心肌细胞钙移除机制的适应性, 只有有关 肌质网的报道. 对冬眠状态和活动状态的比较研究显示, 冬眠动物冬眠时心肌细胞肌质网的 体积密度成倍增加, 离体肌质网摄钙功能增强 ${ }^{[14]}$. 最近的报道证明冬眠动物心肌肌质网相 对非冬眠动物也具有适应性 ${ }^{[15]}$. 冬眠动物心肌细胞钲稳态调节是否还存在其他机制尚有待 进一步研究.

本文所发现的冬眠动物保持细胞钲稳态, 避免钲超载的事实, 具有更为广泛的意义. 我 们知道, 心脏的许多病理过程与细胞钲稳态不能有效维持有关. 例如, 在心肌缺血和再灌损 伤中细胞钲超载也是个突出问题 ${ }^{[12]}$ ，相应地，本实验室研究表明黄鼠心肌对缺灌和再灌损伤 具有较强的耐受性. 如果冬眠动物的某些适应性生理机理能为医学所借鉴, 会对治疗某些疾 病 增进人体健康提供新思路. 因此我们认为, 进一步深入认识冬眠动物细胞钙稳态机制对 有关医学问题有潜在的指导意义.

致谢 本文实验在中国农业科学院农业部蔬菜生理与遗传重点开放实验室完成, 得到该 室同志们的热情帮助，特此致谢.

\section{参考文献}

1 Wang S Q, Huang Y H, Zhou Z Q. Dependence of myocardial hypothermia resistance on sources of activator calcium. Cryobiol, 1997, 35: $193 \sim 200$

2 Liu B, Arlock P, Wohlfart B, et al. Temperature effects on the Na and Ca currents in rat and hedgehog ventricular muscle. Cryobiol, 1991, 28: 96 104

3 Lattanzio F A Jr. The effects of $\mathrm{pH}$ and temperature on fluorescent calcium indicators as determined with Chelex-100 and EDTA buffer systems. Biochem Biophys Res Commun, 1990, 171: 102 108

4 Reeves R B. An imidazole alpha stat hypothesis for vertebrate acid base regulation: tissue carbon dioxide content and body temperature in bullfrogs. Respir Physiol, 1972, 14: 219 236

5 Swain J A, McDonald T J Jr, Robbins R C, et al. Relationship of cerebral and myocardial intracellular $\mathrm{pH}$ to blood $\mathrm{pH}$ during hypothermia. Am J Physiol, 1991, 260: H 1 640 1644

6 Liu B, Wang L C H, Belke D D. Effect of low temperature on the cytosolic free $\mathrm{Ca}^{2+}$ in rat ventricular myocytes. Cell Calcium, 1991, 12: $11 \sim 18$

7 Grynkiewicz G, Poenie M, Tien R Y. A new generation of $\mathrm{Ca}^{2+}$ indicators with greatly improved fluorescent properties. J Biol Chem, 1985, 260: 3 440 3450

8 Harrison S M, Bers D M. Correction of proton and Ca association constants of EGTA for temperature and ionic strength. Am J Physiol, 1989. 256: C1 250 1256

9 Baker A J, Brandes R, Schreur J H, et al., Protein and acidosis alter calcium-binding and fluorescence spectra of the calcium indicator indor 1. Biophys J, 1994, 67: 1 646 1654

10 Hove-Madsen L, Bers D M. Indo 1 binding to protein in permeabilized ventricular myocytes alters its spectral and Ca binding properties. Biophys J, 1992, 63: 89 97

11 Cheng H, Lederer W J, Cannell M B. Calcium sparks: elementary events underlying excitation contraction coupling in heart muscle. Science, 1993, 262: 740 744

12 Tani M. Mechanisms of calcium overload in reperfused ischemic myocardium. Ann Rev Physiol, 1990, 52: 543 559

13 Lakatta E G. Functional implications of spontaneouse sarcoplasmic reticulum $\mathrm{Ca}^{2+}$ release in the heart. Cardiovasc Res, 1992, 26: 193 214

14 唐亚俊，王世强，周曾铨. 黄鼠心肌肌质网超微结构和摄钲速率的季节性变化. 生理学报，1995，47: 478 484

15 Liu B, Belke D D, Wang L C H. $\mathrm{Ca}^{2+}$ uptake by cardiac sarcoplasmic reticulun at low temperature in rat and ground squirrel. Am J Physiol, 1997, 272: R1 121 1127 